\title{
Suppressor of cytokine signaling-3 is a biomechanical stress-inducible gene that suppresses gp130-mediated cardiac myocyte hypertrophy and survival pathways
}

\author{
Hideo Yasukawa, ${ }^{1}$ Masahiko Hoshijima, ${ }^{1}$ Yusu Gu, ${ }^{1}$ Tomoyuki Nakamura, ${ }^{1}$ \\ Sylvain Pradervand, ${ }^{1}$ Toshikatsu Hanada, ${ }^{2,3}$ Yasushi Hanakawa, ${ }^{4}$ Akihiko Yoshimura, ${ }^{2}$ \\ John Ross, Jr., ${ }^{1}$ and Kenneth R. Chien ${ }^{1}$

\begin{abstract}
${ }^{1}$ University of California San Diego (UCSD)-Salk Program in Molecular Medicine, UCSD Institute of Molecular Medicine, and Department of Medicine, University of California San Diego, La Jolla, California, USA

${ }^{2}$ Division of Molecular and Cellular Immunology, Medical Institute of Bioregulation, Kyushu University, Fukuoka, Japan

${ }^{3}$ Division of Molecular Genetics, Institute of Life Science, Kurume University, Kurume, Japan

${ }^{4}$ Department of Dermatology, Ehime University School of Medicine, Ehime, Japan
\end{abstract}

Address correspondence to: Kenneth R. Chien, UCSD Institute of Molecular Medicine, University of California San Diego, La Jolla, California 92093-0613C, USA. Phone: (858) 534-6835; Fax: (858) 534-8081; E-mail: kchien@ucsd.edu.

Received for publication August 9, 2001, and accepted in revised form October 1, 2001.

The gp130 cytokine receptor activates a cardiomyocyte survival pathway during the transition to heart failure following the biomechanical stress of pressure overload. Although gp130 activation is observed transiently during transverse aortic constriction (TAC), its mechanism of inactivation is largely unknown in cardiomyocytes. We show here that suppressor of cytokine signaling 3 (SOCS3), an intrinsic inhibitor of JAK, shows biphasic induction in response to TAC. The induction of SOCS3 was closely correlated with STAT3 phosphorylation, as well as the activation of an embryonic gene program, suggesting that cardiac gp130-JAK signaling is precisely controlled by this endogenous suppressor. In addition to its cytoprotective action, gp130-dependent signaling induces cardiomyocyte hypertrophy. Adenovirus-mediated gene transfer of SOCS3 to ventricular cardiomyocytes completely suppressed both hypertrophy and antiapoptotic phenotypes induced by leukemia inhibitory factor (LIF). To our knowledge, this is the first clear evidence that these two separate cardiomyocyte phenotypes induced by gP130 activation lie downstream of JAK. Three independent signaling pathways, STAT3, MEK1ERK1/2, and AKT activation, that are coinduced by LIF stimulation were completely suppressed by SOCS3 overexpression. We conclude that SOCS3 is a mechanical stress-inducible gene in cardiac muscle cells and that it directly modulates stress-induced gp130 cytokine receptor signaling as the key molecular switch for a negative feedback circuit for both myocyte hypertrophy and survival.

J. Clin. Invest. 108:1459-1467 (2001). DOI:10.1172/JCI200113939.

\section{Introduction}

Heart failure is a leading cause of mortality that ensues following the chronic activation of biomechanical stress pathways resulting from various forms of myocardial injury (1-3). The central challenge in heart failure is the determination of the molecular mechanisms by which compensatory hypertrophy can ultimately lead to an irreversible decompensation in cardiac function. The common receptor component of the IL-6 family of cytokines, gp130, has been demonstrated to play an important role in cardiac hypertrophy and heart failure (4-6). The gp130 cytokines, such as cardiotrophin-1 (CT-1) and leukemia inhibitory factor (LIF), are potent inducers of cardiomyocyte hypertrophy $(7,8)$ and also serve as myocyte survival factors (9). In the heart, CT-1 and LIF are induced by the biomechanical stress of mechanical stretch or aortic banding $(10,11)$, and recent clinical studies have documented elevated levels of CT- 1 in patients with congestive heart failure (12). In addition, mice that harbor a ventricularrestricted knockout of gp130 display a rapid-onset dilated cardiomyopathy and massive myocyte apoptosis during the biomechanical stress associated with transverse aortic constriction (TAC) (4).

Binding of ligands to the gp130 and LIF receptor complex triggers dimerization and results in the activation of janus kinase (JAK). In turn, activated JAKs rapidly phosphorylate tyrosine residues of these receptors and subsequent recruitment of various signaling molecules including signal transducer and activator of transcription 3 (STAT3) to the receptor complex. Activated STAT3 dimers translocate to the nucleus, where they lead to transcriptional activation of downstream target genes $(13,14)$. Although the activation of JAKmediated gp130 signaling pathways in pressure overload has been relatively well described, little is known about the negative feedback mechanisms that must terminate the activation of the pathway to prevent hyperstimulation by gp130 cytokines, which may have independent pathological effects on cardiac function.

In this regard, the pathways that direct myocyte survival and hypertrophy following gp130 stimulation must be 
tightly controlled to ensure the appropriate duration and intensity of the action of gp130 cytokines. The JAK signaling pathway is regulated by several mechanisms, including receptor internalization, dephosphorylation by protein phosphatases, and degradation by the proteasome pathway. Three groups have independently identified the suppressor of cytokine signaling (SOCS) family (also referred to as cytokine-inducible SH2 protein [CIS] or STAT-induced STAT inhibitor [SSI] family) proteins as cytokine-inducible inhibitors of cytokine signaling (15-17). The eight members (CIS and SOCS1-7) of this family are characterized structurally by a variable $\mathrm{N}$-terminal region, a central SH2 domain and a C-terminal SOCS box motif (18-20). Functionally, CIS and SOCS1-3 proteins interact with cytokine receptors and/or JAKs, thereby inhibiting activation of kinases and STATs. SOCS3 is induced by a variety of cytokines, including CT1 and LIF (21-23). SOCS3 binds to JAKs via cytokine receptor, thereby inhibiting the cytokine receptor signaling $(24,25)$. SOCS3-deficient mice are embryonic-lethal through the marked erythrocytosis (26), preventing the analysis of the cardiac phenotype in SOCS3 knockout mice. To investigate the role of SOCS3 in cardiac hypertrophy, we examined the induction of SOCS3 in heart and its relationship to gp130 signaling pathway using a well-characterized mouse model of TAC.

In this study, we found that SOCS3 is markedly induced not only during acute response phase, but also during hypertrophic response phase following TAC, and this late-phase SOCS3 induction is closely correlated with embryonic gene activation during TAC. These results suggest that there is an important link between SOCS3 induction and cardiac hypertrophy. We also found that forced expression of SOCS3 completely suppressed the ability of LIF and CT-1 to induce hypertrophy and antiapoptosis in cardiomyocytes. These findings suggest that SOCS3 has an important role in the heart, where it acts as a negative feedback regulator of JAK-mediated gp130 signaling pathways. Accordingly, these studies identify SOCS3 as a new potential therapeutic target in heart failure, whereby the promotion of myocyte survival might be attained via the inhibition of this negative regulator of the inducible gp130 myocyte survival pathway.

\section{Methods}

TAC surgery. Transverse thoracic aortic constriction was performed as described previously (27) on 8-week-old adult mice (C57BL/6×SJL; The Jackson Laboratory, Bar Harbor, Maine, USA). At an acute phase (15 minutes to 3 hours after banding) and hypertrophic phase (2-14 days after banding) after surgery, animals from the experimental and sham-operated groups were sacrificed and the hearts were removed. Left ventricles were weighed and quickly frozen in liquid nitrogen for total RNA and protein extraction.

Recombinant adenoviruses. Because of the toxic effect on 293 cells used for recombinant virus production, CreLoxP conditional expression system was employed to generate adenovirus vectors following the protocol described by Kanegae et al. (28). Adenovirus vectors containing the genes for LacZ (AxCALacZ), myc-tagged SOCS3 (AxCASOCS3), myc-tagged CIS (AxLNLCIS), myc-tagged SOCS1 (AxLNLSOCS1), and Cre recombinase (AxCANCre) were prepared via homologous recombination in 293 cells, as described previously (29).

Cardiomyocyte culture and adenoviral infection. Cardiomyocytes were prepared using a Percoll gradient method as described previously (30). Myocytes from 1to 2-day-old Sprague-Dawley rats were plated in serumcontaining medium overnight. Subsequently, the cells were changed into low serum, and infected with recombinant adenoviruses at an moi of 5-10 viral particles per cell for 8 hours. The cells were then cultured in serum-free medium for an additional 24 hours before morphological or biochemical analysis.

Immunocytochemical assay. Cells were fixed in 3.7\% formaldehyde and permeabilized in $0.3 \%$ Triton X-100. The atrial natriuretic factor (ANF) protein was detected using rabbit anti-rat ANF polyclonal antibody (Peninsula Laboratories Inc., San Carlos, California, USA) and FITC-conjugated goat anti-rabbit secondary antibody. The F-actin was detected using TRITC-conjugated phalloidin (Sigma Chemical Co., St. Louis, Missouri, USA) (31). Experiments were performed in triplicate and repeated at least three times.

Cell survival assay and apoptosis analysis. Cell survival was analyzed using the 3-(4,5-dimethylthiaziazol-2-yl)2,5diphenyl tetrazolium bromide (MTT) method as reported previously (9). For analysis of apoptotic cells, a DNA fragmentation assay was performed as described previously using a DNA isolation kit (Promega Corp., Madison, Wisconsin, USA) and standard agarose gel electrophoresis (9). Fragmented and condensed nuclei in apoptotic cells were also identified by TUNEL assays and by staining with DAPI dye as described previously $(9,30)$. All of the experiments were performed in triplicate and repeated at least three times.

Immunoblot and immunoprecipitation analysis. The left ventricles were homogenized in lysis buffer containing $50 \mathrm{mM}$ Tris- $\mathrm{HCl}$ ( $\mathrm{pH} 7.5$ ), $1 \% \mathrm{NP}-40,150 \mathrm{mM} \mathrm{NaCl}$, $10 \%$ glycerol, $1 \mathrm{mM}$ sodium orthovanadate, and protease inhibitor cocktail (Sigma Chemical Co.). The total cell extracts were resolved by SDS-PAGE, and proteins were detected by immunoblotting as described (18). Anti-ERK1/2, anti-MEK, anti-p38, anti-AKT, antiSTAT3, anti-JAK1, anti-gp130, anti-phosphotyrosine, and phosphospecific antibodies were purchased from New England Biolabs Inc. (Beverly, Massachusetts, USA). Immunoprecipitation with anti-JAK1 and antigp130 was performed as described previously $(23,24)$.

Statistical analysis. Analyses between two groups were performed using unpaired two-tailed $t$ tests, with $P$ values less than 0.01 considered as significantly different.

\section{Results}

Induction of SOCS3 by mechanical stress in in vivo myocardium. Since SOCS3 and SOCS1 are induced by gp130- 
STAT3 signaling, we investigated how SOCS3 and SOCS1 are implicated in cardiac hypertrophy during in vivo pressure overload. Two days and 7 days of TAC in wild-type mice resulted in $6 \%$ and $65 \%$ increase in leftventricular-to-body-weight ratio, respectively, compared with sham-operated mice (Figure 1a). SOCS3 was highly induced not only during the acute response phase, but also during the hypertrophic response phase following TAC (Figure 1b). Interestingly, the second peak of SOCS3 expression was consistent with the onset of cardiac hypertrophy and correlated well with the activation of ANF and brain natriuretic peptide (BNP) genes (Figure 1, $c$ and d). In contrast, SOCS1 mRNA induction remained at undetectable levels in the pressure-overloaded heart (data not shown). These results suggest the possibility of an important link between SOCS3 induction and cardiac hypertrophy during in vivo pressure overload.

Transient activation of gp130 signaling during pressure overload induced myocardial bypertrophy. In our previous study as well as from results of other groups $(4,7)$, it has become clear that there is transient activation of JAKSTAT signaling after pressure overload. In the present study, we have analyzed both the acute (15 minutes to 3 hours) and the hypertrophic (2-14 days) phases of mechanical stress-induced signaling following TAC. As shown in Figure 2, we now provide clear evidence that STAT3 phosphorylation is associated with the expression of SOCS3 with two sharp activation peaks at 3 hours and 2 days following TAC. We also evaluated the activation of other gp130 downstream molecules including ERK1/2, p38, and AKT during TAC. ERK and p38 also displayed a pattern of biphasic induction. The first phase of ERK activation was initiated 15 minutes after TAC and was sustained over 3 hours, while p38 showed a shorter duration of activation during the first phase. Both ERK and p38 phosphorylations were subsequently re-elevated at 2 days following TAC, and this activation was sustained for 7 days. On the other hand, activation of AKT was observed predominantly at the late phase and continued for 14 days following TAC. SOCS3 mRNA and protein is induced in the heart by in vivo infusion of gp130 cytokines (23). In addition, recent studies have revealed that the SOCS3 promoter contains a functionally important STAT-binding element (21). Collectively, our data suggest that TAC-induced cardiac gp130-JAK-STAT3 signaling is under a tight negative feedback loop via SOCS3. SOCS3 is highly expressed in cardiac myocytes in response to gp130 cytokines. To confirm that SOCS family members are induced in a pure population of cardiomyocytes, we examined the induction of SOCS1 and SOCS3 mRNA following exposure to LIF in cultured ventricular cardiomyocytes. SOCS3 was markedly induced by LIF in cardiomyocytes (Figure 3a). SOCS3 was acutely induced by LIF with a sharp peak that endured less than 1 hour, and this was sustained at a lower level for 24 hours. We also examined the effect of other cytokines and growth factors on SOCS3 and SOCS1 induction in cardiomyocytes. Another gp130 cytokine,
CT-1, also induced high levels of SOCS3, whereas IFN- $\gamma$, angiotensin II (Ang II), and neuregulin (NRG) only marginally induced SOCS3 (Figure $3 \mathrm{~b}$ ). SOCS1 was only induced by IFN- $\gamma$ (Figure 3b). Thus, gp130 cytokines specifically induced SOCS3, while IFN- $\gamma$ induced SOCS1 in cardiac myocytes.

SOCS3 and SOCS1 inhibit the CT-1-induced hypertrophic response. We examined the effect of the forced expression of SOCS genes on the biological action of gp 130 cytokines in cardiomyocytes using recombinant adenoviruses. First, we examined the effect of SOCSs on CT-1-induced cardiac myocyte hypertrophy. CT-1-induced hypertrophy was not inhibited in cardiomyocytes infected with adenoviruses expressing either LacZ (Figure 4, a and e) or CIS (Figure 4, $d$ and h), which suppresses STAT5 but not STAT3 signaling

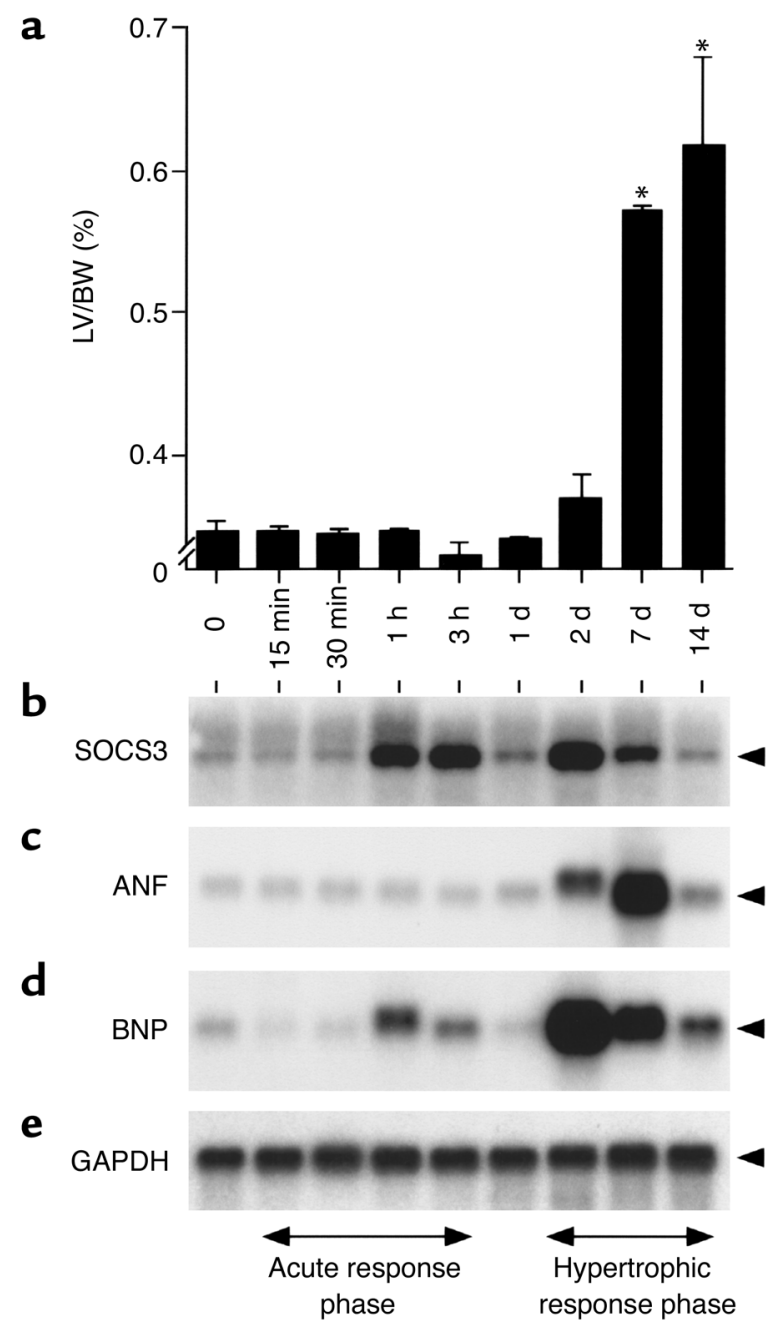

Figure 1

Correlation of SOCS3 induction and embryonic gene activation during TAC in wild-type mice. (a) Left ventricular weight to body weight (LV/BW) was measured in mice during TAC $(n=3-4)$. Results are shown as means $\pm \mathrm{SD}$. ${ }^{*} P<0.01 \mathrm{vs}$. sham. Total RNA was prepared from murine left ventricles at the indicated time afterTAC. Total RNA was blotted with probe for (b) SOCS3, (c) ANF, (d) BNP, and (e) GAPDH. Representative Northern blots from three independent experiments are shown. 


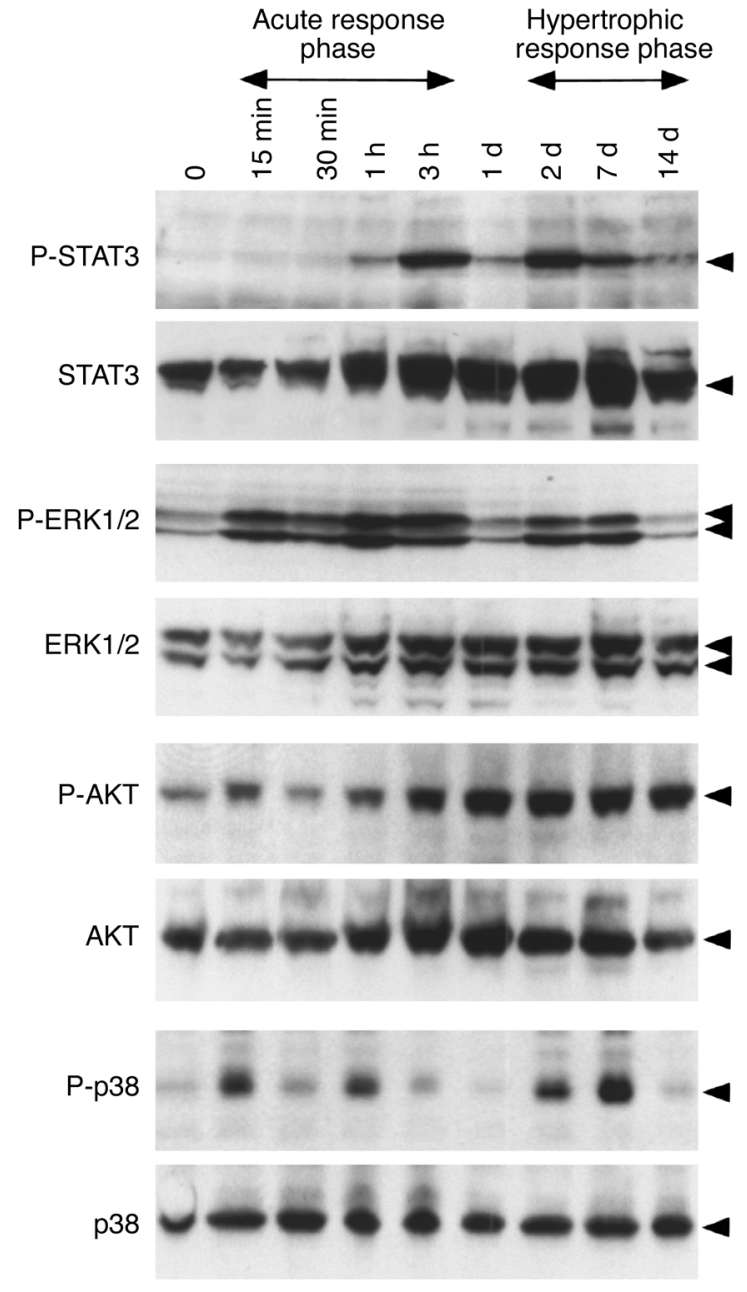

(17). In contrast, infection with viruses that expressed either SOCS3 (Figure 4, b and f) or SOCS1 (Figure 4, c and g) completely inhibited CT-1-induced myocyte hypertrophy. A quantitative evaluation of these results is shown in Figure 4i. Expression levels of the myctagged CIS, SOCS1, and SOCS3 were confirmed by the anti-myc immunoblotting (Figure 4j). We also demonstrated that expression of the hypertrophic marker ANF was inhibited in almost all cardiac myocytes expressing ectopic SOCS1 (Figure 5, i and k) or SOCS3 (Figure 5, e and g), but not in cells expressing ectopic LacZ (Figure 5, a and c) or CIS (Figure 5, $\mathrm{m}$ and o). Actin polymerization in cardiomyocytes was also visualized by phalloidin staining. SOCS1 (Figure 5, $\mathrm{j}$ and 1) and SOCS3 (Figure 5, f and h) significantly inhibited sarcomeric organization. Thus, forced expression of

\section{Figure 3}

gp130 cytokine-dependent induction of SOCS1 and SOCS3 mRNA in cardiomyocytes. (a) Myocytes were stimulated with $10 \mathrm{ng} / \mathrm{ml}$ LIF for the indicated time, and total RNA was blotted with SOCS3 and SOCS1 probes from mouse full-length cDNA. (b) Ventricular myocytes were stimulated with the indicated cytokines or growth factors for 1 hour, and total RNA was blotted with SOCS3 and SOCS1 probes. The experiments were repeated three times, yielding similar results.

\section{Figure 2}

Transient activation of gp130 downstream signaling during TAC. The total cell lysate was prepared from murine left ventricles at the indicated time after TAC, and blotted with phospho-STAT3 (P-STAT3), STAT3, phospho-ERK1/2 (P-ERK1/2), ERK1/2, phospho-AKT (P-AKT), AKT, phospho-p38 (P-p38), and p38 antibodies. Representative Western blots from three independent experiments are shown.

SOCS1 and SOCS3 inhibited phenotypic features of cardiomyocyte hypertrophy that occur in direct response to gp130 cytokines.

SOCS3 and SOCS1 block the antiapoptotic action of LIF. As it has been shown that CT-1 and LIF promote cardiac myocyte survival, we examined the effect of SOCSs on the antiapoptotic action of LIF. We first performed DNA fragmentation assays to detect the presence of internucleosomal laddering in genomic DNA. DNA fragmentation in myocytes was observed 2 days after serum deprivation (Figure 6a, lanes 1, 3, 5 , and 7). LIF suppressed DNA fragmentation of myocytes expressing LacZ and CIS (Figure 6a, lanes 2 and 8). In comparison, LIF did not inhibit DNA fragmentation of myocytes expressing ectopic SOCS3 and SOCS1 (Figure 6a, lanes 4 and 6). Ventricular myocytes undergoing apoptosis were also analyzed by TUNEL staining and by nuclear staining with DAPI dye. Two days after serum deprivation, chromosomal condensation and fragmentation of nuclei were observed in a high percentage of LIF-treated myocytes expressing SOCS3 (Figure 6, h and i) and SOCS1 (Figure 6,1 and $\mathrm{m}$ ). A quantitative evaluation of these results is shown in Figure 6r. We also evaluated the effects of SOCS genes on the survival of cardiac myocytes promoted by LIF. SOCS3 and SOCS1 were

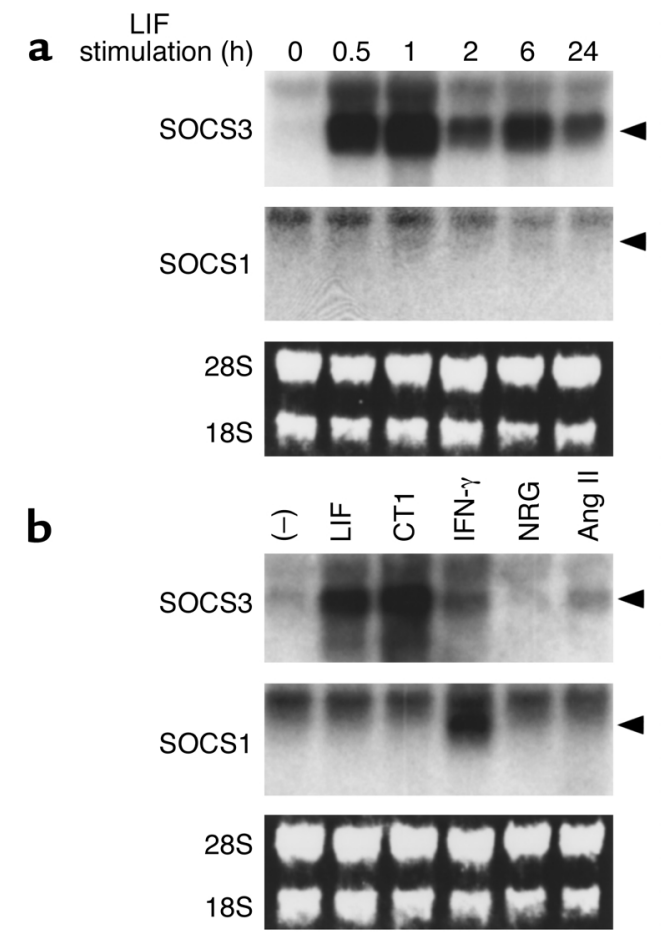



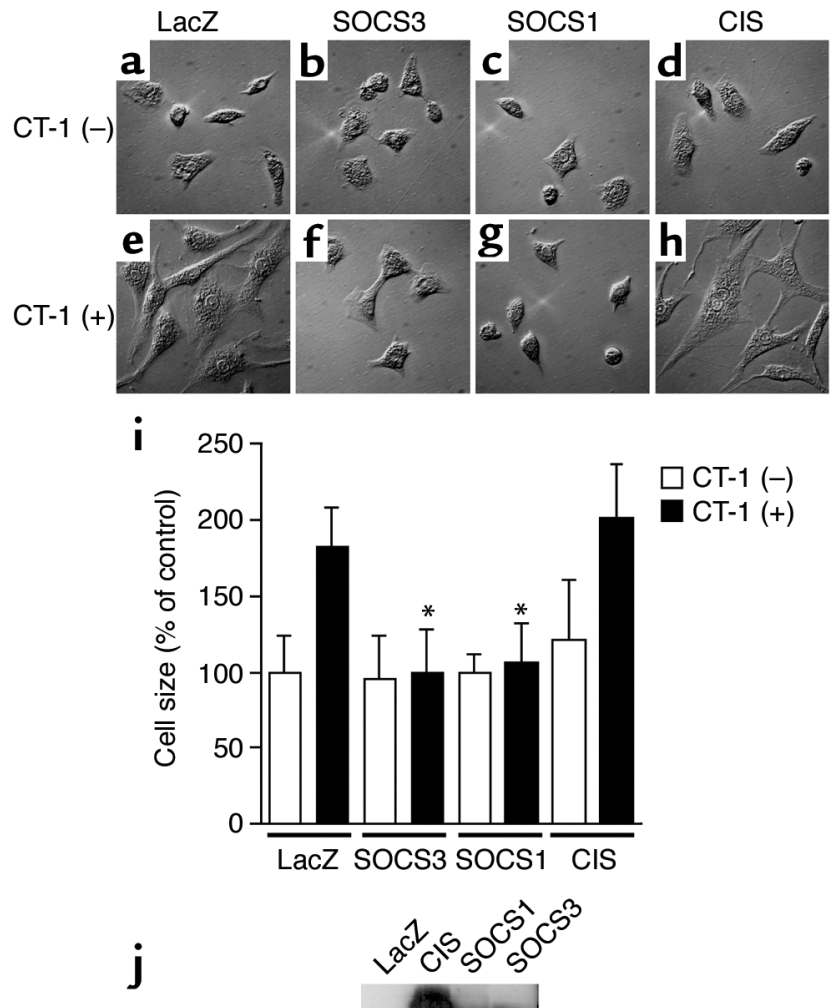

Blot: $\alpha-m y c$

capable of blocking cell survival promoted by LIF (Figure 7). Thus, SOCS3 and SOCS1 blocked the antiapoptotic action of LIF, suggesting that SOCS3 and SOCS1 negatively regulate LIF activation of cardiac myocyte survival pathways.

SOCS3 suppresses multiple gp130 downstream signaling pathways. To elucidate the potential mechanism whereby SOCS3 inhibits gp130 cytokine-mediated cell hypertrophy and cell survival, we examined the effect of SOCS3 on the LIF-induced activation of STAT3, MEK1, ERK1/2, and AKT, which are known to be involved in various steps of cardiac hypertrophy and myocyte survival $(5,6,9,32)$. Cardiac myocytes expressing ectopic SOCS3 were stimulated with or without LIF, and cell extracts were blotted with phosphorylation-specific antibodies to STAT3, MEK1, ERK1/2, and AKT. These signaling molecules were rapidly activated by LIF in cardiomyocytes expressing LacZ, but their

\section{Figure 5}

Suppression of CT-1-induced ANF expression and sarcomere organization by SOCS3 and SOCS1. Myocytes were infected with virus vectors and maintained in the serum-depleted medium for 24 hours. Cells were stimulated with or without $1 \mathrm{nM} \mathrm{CT}-1$ for 52 hours. Cells were stained for ANF protein ( $\mathbf{a}, \mathbf{c}, \mathbf{e}, \mathbf{g}, \mathbf{i}, \mathbf{k}, \mathbf{m}$, and $\mathbf{o})$ and for F-actin using TRITC-conjugated phalloidin ( $\mathbf{b}, \mathbf{d}, \mathbf{f}, \mathbf{h}, \mathbf{j}, \mathbf{l}, \mathbf{n}$, and $\mathbf{p})$. Induction of ANF expression was identified from positive perinuclear staining of ANF protein. (q) Percentage of myocytes expressing ANF protein was quantified. The experiments were repeated three times. Results are shown as means \pm SD. ${ }^{*} P<0.01$ vs. LacZ with CT-1 $(n=100$ cells each $)$.

\section{Figure 4}

Suppression of CT-1-induced cardiomyocyte hypertrophy by SOCS3 and SOCS1. (a-h) Myocytes were infected with Ad-LacZ, Ad-SOCS3, Ad-SOCS1, or Ad-CIS with Ad-Cre and maintained in serum-depleted medium for 24 hours. Cells were stimulated without (a-d) or with 1 nM CT-1 (e-h) for 72 hours. Cells were examined under light microscopy, and (i) the cell surface areas were analyzed using NIH Image software (NIH, Bethesda, Maryland, USA) as a relative percentage of control CT-1 minus LacZ-infected cardiomyocytes. A total of 300 cells were examined for each measurement. Results are shown as means \pm SD. ${ }^{*} P<0.01 \mathrm{vs}$. LacZ with CT-1. (j) Expression levels of CIS/SOCSs were confirmed by an anti-myc blotting.

activation was completely inhibited in cardiomyocytes expressing SOCS3 (Figure 8a). Thus, SOCS3 suppressed multiple gp130 downstream signaling pathways. To further investigate the inhibitory mechanisms of SOCS3 on gp130 signaling, we examined SOCS3 interaction with JAK1 and gp130 in cardiomyocytes. As shown in Figure 8b, JAK1 and gp130 were coimmunoprecipitated with SOCS3, and SOCS3 inhibited phosphorylation of gp130 as well as JAK1. Thus, the suppression of the STAT3, MEK1-ERK1/2, and AKT pathways by SOCS3 can be explained by the suppression of JAK kinases, which occurs by direct interaction of SOCS3 with a gp130-JAK1 complex.
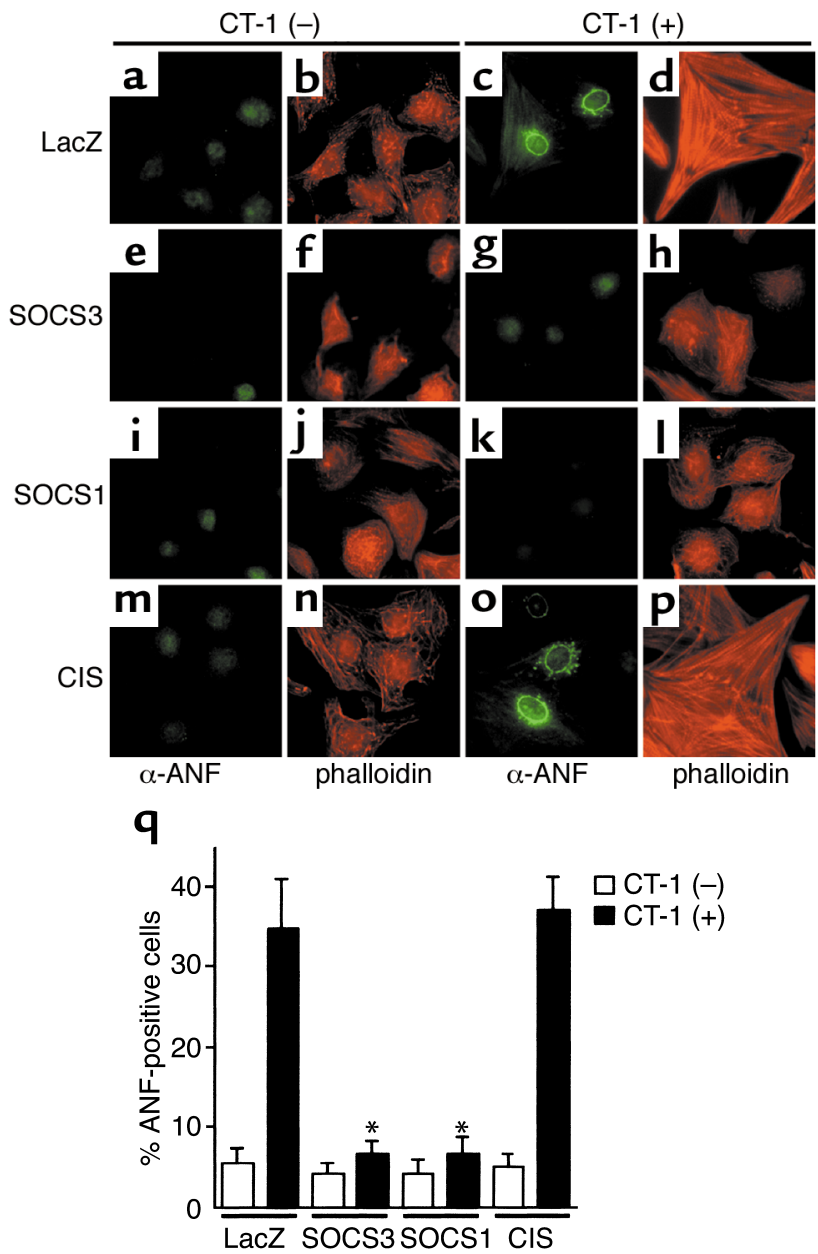
a

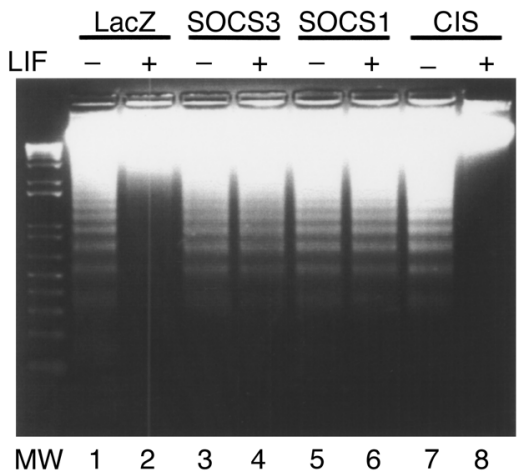

$r$

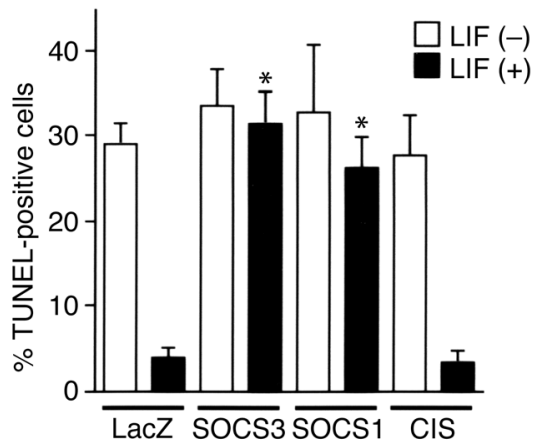

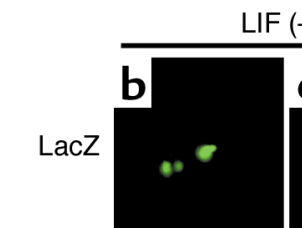

IF (-)
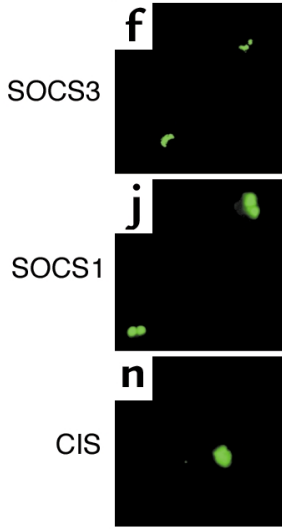

TUNEL
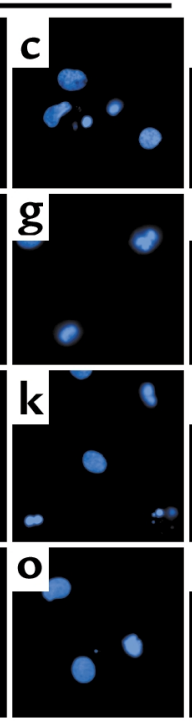

DAPI

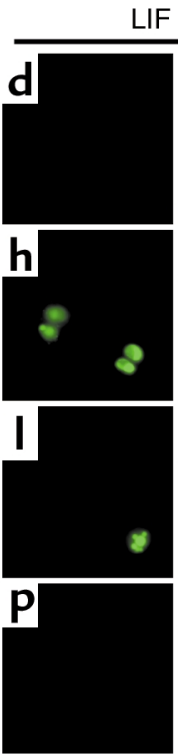

TUNEL
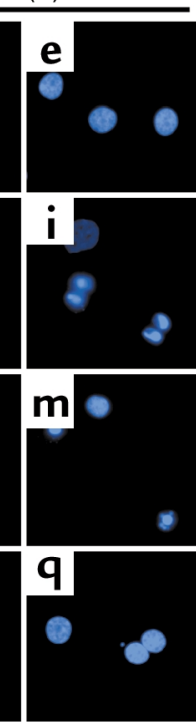

DAPI

\section{Figure 6}

SOCS1 and SOCS3 block the antiapoptotic action promoted by LIF. Myocytes were infected with viruses and stimulated with or without 10 $\mathrm{ng} / \mathrm{ml}$ LIF for 48 hours. LIF inhibited cardiomyocyte apoptosis induced by serum deprivation as reported previously (9). (a) Genomic DNA was extracted and electrophoresis was performed on a $2 \%$ agarose gel. MW, $1 \mathrm{~kb}$ plus molecular weight marker. (b-q) Cells were stained using TUNEL methods and DAPI dye, as described in Methods. Green fluorescence shows terminal deoxynucleotidyl transferase-labeled nuclei. ( $\mathbf{r}$ ) Quantitative analysis of percentage of cells undergoing apoptosis as measured by TUNEL staining $(n=100$ cells each). The experiments were repeated three times. Results are shown as means \pm SD. ${ }^{*} P<0.01$ vs. LacZ with LIF.

\section{Discussion}

In the present study, we focused on the role of the negative cytokine regulator SOCS3 in cardiac hypertrophy and cell survival using an in vivo murine model of pressure overload and an in vitro model based on neonatal rat cardiomyocytes. We found that SOCS3 was highly induced not only during the acute response phase, but also during the hypertrophic response phase following TAC. Interestingly, the second peak of SOCS3 expression was consistent with the onset of cardiac hypertrophy 2 days after TAC. In addition, the second phase of SOCS3 induction was closely correlated with the activation of ANF and BNP genes during TAC. These results suggest that there is an important link between SOCS3 induction and in vivo cardiac hypertrophy. We also demonstrated a negative effect of SOCS3 on gp130-JAK signaling and cardiomyocyte hypertrophy. Thus, during the progression of cardiac hypertrophy, SOCS3 participates in a negative feedback loop switching off the gp130 signaling cascade. These findings suggest that inhibition of gp130-JAK signaling by SOCS3 may ensure the termination of the cardiac hypertrophic response.

\section{Figure 7}

Blockade of LIF-dependent cardiac muscle cell survival by SOCS1 and SOCS3. Myocytes treated with viral vectors were plated in 24-well plates and maintained for 3 days (a) or 7 days (b) in serum-free medium with or without LIF $(10 \mathrm{ng} / \mathrm{ml})$. Cell viability was measured using MTT assays. The experiments were repeated five times. Results are shown as means $\pm \mathrm{SD}$ $(n=3)$. ${ }^{*} P<0.01$ vs. LacZ with LIF.
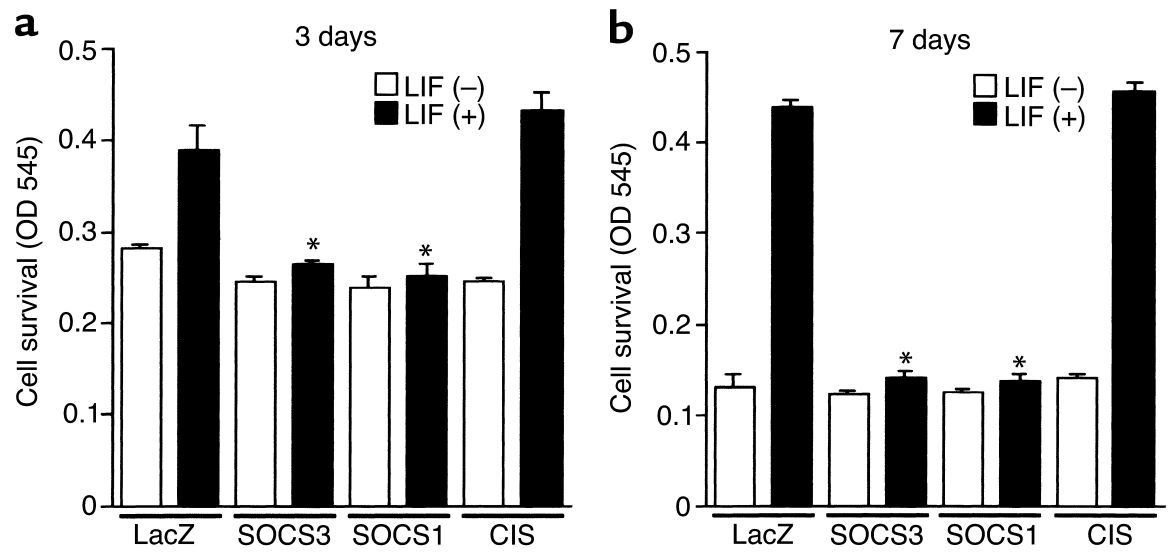
Like SOCS3, SOCS1 also suppresses gp130-mediated myocyte hypertrophy and survival in vitro (Figures 4-7). However, the effect of SOCS1 on gp130 signaling may not be in a physiological negative feedback loop, as gp130 cytokines did not induce SOCS1 in cardiomyocytes and SOCS1 is not induced in the pressure-overloaded heart. As shown in gene knockout studies of SOCS1 and SOCS3, their expression patterns reflect their physiological function as part of negative feedback regulators $(26$, $33,34)$. SOCS1 is strongly induced by IFN- $\gamma$, and SOCS1 is an essential modulator of IFN- $\gamma$ effects in vivo $(22,33$, 34). In this study, we found that SOCS1 is specifically induced by IFN- $\gamma$ in cardiomyocytes (Figure 3). Since IFN- $\gamma$ is essential for host defense, cardiac SOCS1 may play a particularly important role in pathological states such as virally induced heart disease.

STAT3 has been shown to play an important role in transducing both hypertrophic and cytoprotective signals in vitro and in vivo $(6,7,10,13,34,35)$. In this study, we found that SOCS3 induction was closely correlated with the activation of STAT3 during TAC, which most likely reflects previous findings that the SOCS3 promoter contains a functionally important STAT3-binding element (21). Thus, in addition to its hypertrophic and cytoprotective effects, STAT3 could activate negative feedback loop of the gp130 pathway via the induction of SOCS3. We hereby propose a model for the negative regulation of mechanical stress-induced cardiac hypertrophy via gp130 cytokine receptor signaling (Figure 9). Biomechanical stress activates the JAK-mediated gp130 cascade. SOCS3 is induced in myocardium in a STAT3-dependent manner. SOCS3 suppresses JAK kinase activity by binding to both gp130 and JAKs. By inhibiting JAKs, SOCS3 negatively regulates stress-induced gp130 activation and cardiac hypertrophy. It is likely that this negative regulatory circuit serves to prevent hyperstimulation by gp130 cytokines, which may have independent pathological effects on cardiac function. In other words, the delicate balance between the activation of gp130-JAK signaling and the induction of its negative feedback regulator SOCS3 might be important in the transition between cardiac hypertrophy and failure. a

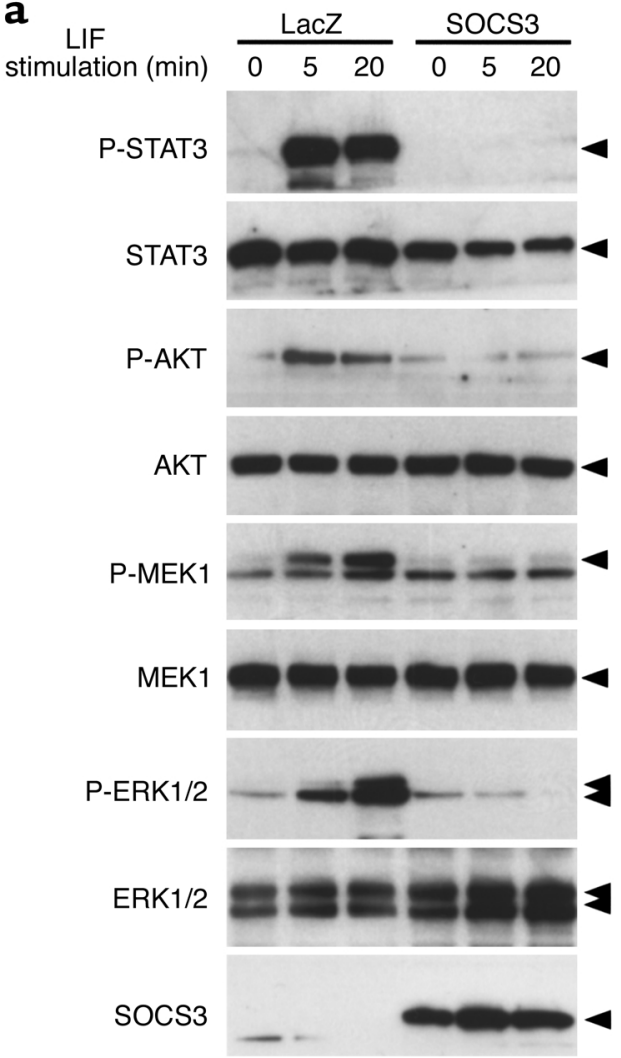

b

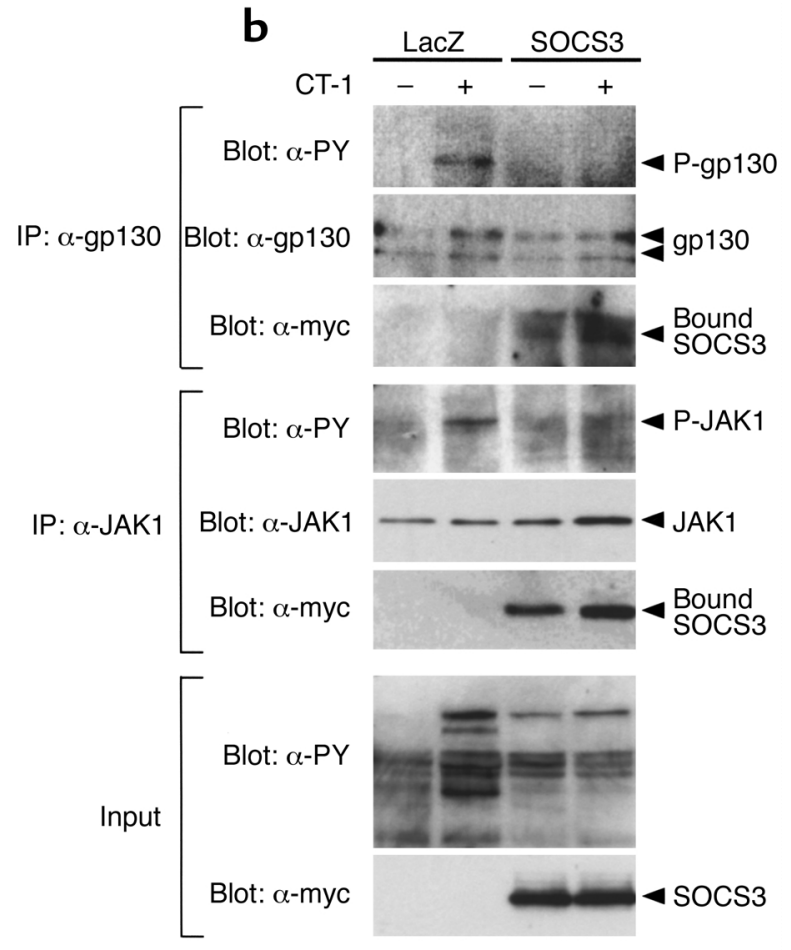

\section{Figure 8}

(a) Inhibition of multiple gp130 downstream signaling by SOCS3. Myocytes were treated with Ad-LacZ or Ad-SOCS3, serum-depleted for 24 hours, and then stimulated with $10 \mathrm{ng} / \mathrm{ml}$ LIF for 5 or 20 minutes. Total cell extracts were prepared and blotted with anti-phospho-STAT3 (P-STAT3), anti-STAT3, anti-phospho-AKT (P-AKT), anti-AKT, anti-phospho-MEK1 (P-MEK1), anti-MEK1, anti-phospho-ERK1/2 (P-ERK1/2), and anti-ERK1/2 antibodies. SOCS3 expression was confirmed with an anti-myc antibody. Representative Western blots from three independent experiments are shown. (b) SOCS3 recruitment to activated gp130-JAK1 complex in cardiomyocytes. Myocytes were treated with Ad-LacZ or Ad-SOCS3, serum-depleted for 24 hours, and then stimulated with $1 \mathrm{nM}$ of CT-1 for 10 minutes. Cell extracts were immunoprecipitated with anti-gp130 or anti-JAK1 antibodies. The immunoprecipitates were analyzed by blotting with anti-phosphotyrosine $(\alpha-P Y)$, anti-gp130 ( $\alpha$-gp130), anti-JAK1 ( $\alpha$-JAK1), and anti-myc $(\alpha-$-myc) antibodies. Total cell extracts were also blotted with $\alpha$-PY and $\alpha$-myc antibodies. Data from one experiment are presented. One additional experiment yielded comparable results. 


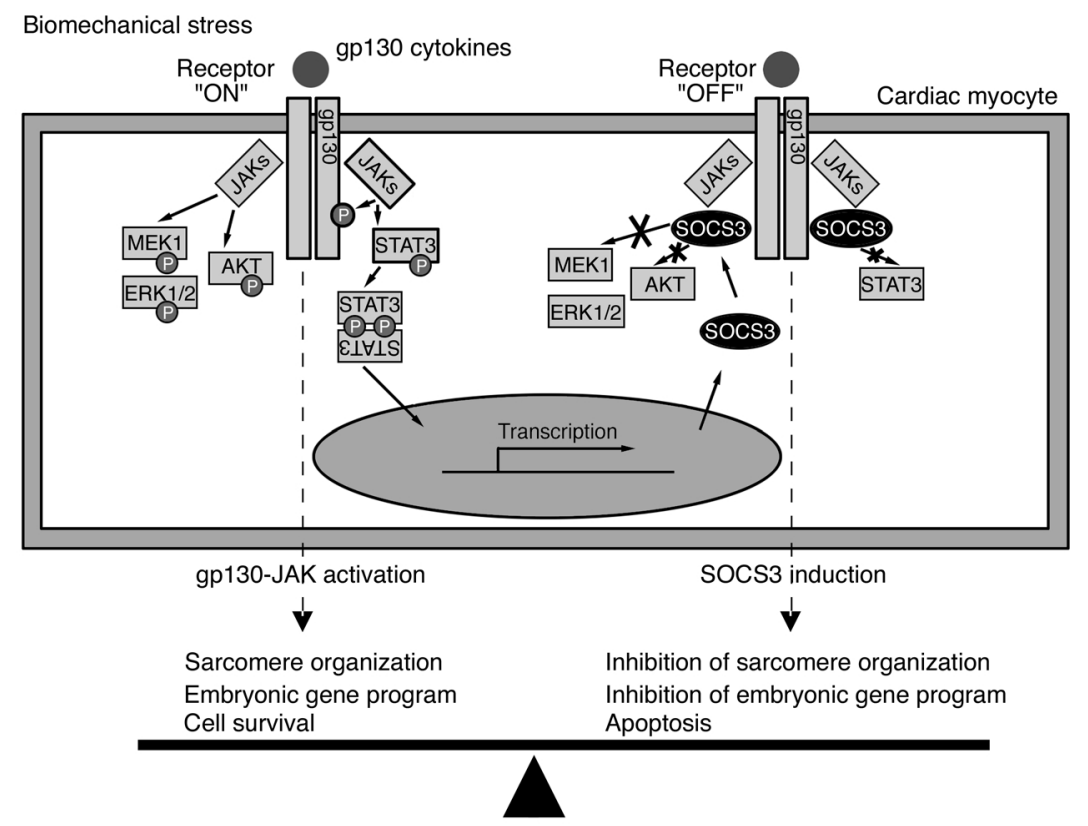

\section{Figure 9}

Working model of the negative feedback circuit for the control of gp130 cytokine receptor signaling during cardiac biomechanical stress. Biomechanical stress activates the JAKmediated gp130 cytokine receptor cascade. SOCS3 is induced in the myocardium in a STAT3-dependent manner, since the SOCS3 promoter has a functionally critical STAT3binding region (21). SOCS3 suppresses JAK kinase activity by binding to JAK-gp130 complex. By inhibiting JAK kinase activity, SOCS3 negatively regulates stress-induced gp 130 activation and multiple downstream signaling pathways. This regulatory circuit serves to counterbalance the gp130 cytokine-induced hypertrophy and cell survival response in cardiomyocytes. Aberrant SOCS3 induction may have independent pathological effects on cardiac function.
Although numerous studies have demonstrated an essential role of intracellular signaling pathways in the regulation of cardiac hypertrophy and failure $(2,3,36)$, there are only a few studies regarding negative feedback regulation of intracellular signaling pathways. Since sustained activation of intracellular signaling may have deleterious effects on cardiac function, the activities of intracellular signaling molecules must be tightly regulated. Recently, Rothermel et al. reported myocyte-enriched calcineurin-interacting protein-1 (MCIP1) that inhibits cardiac hypertrophy by attenuating calcineurin activity (37). MCIP1-5' promoter lesion has dense clustering of NF-AT-binding motifs to mediate the potent response to calcineurin signaling (38), suggesting that MCIP1 participates in the negative feedback loop of calcineurin signaling in myocardium. Bueno et al. reported a negative feedback system for the cardiac mitogen-activated protein (MAP) kinase signaling cascade in which forced expression of MAP kinase phosphatase- 1 negatively regulates the cardiac hypertrophic response by downregulating the three branches of MAP kinases (39). Here, we demonstrated that the endogenous JAK inhibitor SOCS3 was robustly and rapidly induced in LIF-stimulated cardiomyocytes and pressure-overloaded myocardium, and that forced expression of SOCS3 blunted the gp130-mediated cardiomyocyte hypertrophy and survival, suggesting that cardiac SOCS3 modulates gp130 cytokine signaling as a key molecule in a negative regulatory circuit.

These studies raised the possibility that the aberrant action of SOCS3 in the heart might contribute to cardiac diseases that arise through pathological cytokine responses. Further studies of the action of SOCS genes in heart may not only provide new insights into mechanisms of cardiac disease, but also provide a novel set of pharmaceutical targets. In this regard, the inhibi- tion of SOCS3 could represent a novel in vivo strategy to promote gp130-dependent pathways for myocyte survival. The development of mice that harbor a cardiac-restricted ablation of the SOCS3 gene should be informative with regard to the validity of this new therapeutic strategy.

Note added in proof. Recent studies have identified $\mathrm{Kv}$ channel-interacting protein-2 (KCHIP2) as a cytoplasmic $\mathrm{K}$ channel accessory protein that is downregulated during biomechanical stress-induced hypertrophy, and it will become of interest to determine if SOCS3 pathways affect the downregulation of this molecular determinant of malignant arrythmias. (Kuo, H.C., et al. 2001. A defect in the Kv channelinteracting protein 2 (KCHIP) gene leads to a complete loss of the transient outward potassium current (Ito) and confers genetic susceptibility to ventricular tachycardia. Cell. In press.)

\section{Acknowledgments}

We thank Janelle Stricker, Julie Anderson, and Wendy Bardwell for expert assistance, and Sylvia M. Evans for critical review of this manuscript. This study was supported by the NIH, the Jean LeDucq Foundation, and the American Heart Association Endowed Chair (to K.R. Chien). H. Yasukawa is supported by a Banyu Fellowship Award in Cardiovascular Medicine sponsored by Banyu Pharmaceutical Co. and The Merck Company Foundation.

\footnotetext{
1. Hunter, J.J. and Chien, K.R. 1999. Signaling pathways for cardiac hypertrophy and failure. N. Engl. J. Med. 341:1276-1283.

2. Chien, K.R. 1999. Stress pathways and heart failure. Cell. 98:555-558.

3. Sugden, P.H. 1999. Signaling in myocardial hypertrophy: life after calcineurin? Circ. Res. 84:633-646.

4. Hirota, H., et al. 1999. Loss of a gp130 cardiac muscle cell survival pathway is a critical event in the onset of heart failure during biomechanical stress. Cell. 97:189-198.

5. Negoro, S., et al. 2001. Glycoprotein 130 regulates cardiac myocyte survival in doxorubicin-induced apoptosis through phosphatidylinositol
} 
3-kinase/Akt phosphorylation and Bcl-xL/caspase-3 interaction. Circulation. 103:555-561.

6. Uozumi, H., et al. 2001. gp130 plays a critical role in pressure overloadinduced cardiac hypertrophy. J. Biol. Chem. 276:23115-23119.

7. Pan, J., et al. 1998. Involvement of gp130-mediated signaling in pressure overload-induced activation of the JAK/STAT pathway in rodent heart. Heart Vessels. 13:199-208.

8. Wollert, K.C., et al. 1996. Cardiotrophin-1 activates a distinct form of cardiac muscle cell hypertrophy. Assembly of sarcomeric units in series VIA gp130/leukemia inhibitory factor receptor-dependent pathways. $J$. Biol. Chem. 271:9535-9545.

9. Sheng, Z., et al. 1997. Cardiotrophin 1 (CT-1) inhibition of cardiac myocyte apoptosis via a mitogen-activated protein kinase-dependent pathway. Divergence from downstream CT-1 signals for myocardial cell hypertrophy. J. Biol. Chem. 272:5783-5791.

10. Pan, J., et al. 1999. Mechanical stretch activates the JAK/STAT pathway in rat cardiomyocytes. Circ. Res. 84:1127-1136.

11. Wang, F., et al. 2001. Functional significance of hemodynamic overloadinduced expression of leukemia-inhibitory factor in the adult mammalian heart. Circulation. 103:1296-1302.

12. Talwar, S., et al. 2000. Elevated circulating cardiotrophin-1 in heart failure: relationship with parameters of left ventricular systolic dysfunction. Clin. Sci. 99:83-88.

13. Yamauchi-Takihara, K., and Kishimoto, T. 2000. A novel role for STAT3 in cardiac remodeling. Trends Cardiovasc. Med. 10:298-303.

14. Taga, T., and Kishimoto, T. 1997. Gp130 and the interleukin- 6 family of cytokines. Annu. Rev. Immunol. 15:797-819.

15. Naka, T., Fujimoto, M., and Kishimoto, T. 1999. Negative regulation of cytokine signaling: STAT-induced STAT inhibitor. Trends Biochem. Sci. 24:394-398.

16. Nicola, N.A., and Greenhalgh, C.J. 2000. The suppressors of cytokine signaling (SOCS) proteins: important feedback inhibitors of cytokine action. Exp. Hematol. 28:1105-1112.

17. Yasukawa, H., Sasaki, A., and Yoshimura, A. 2000. Negative regulation of cytokine signaling pathways. Annu. Rev. Immunol. 18:143-164.

18. Yasukawa, H., et al. 1999. The JAK-binding protein JAB inhibits Janus tyrosine kinase activity through binding in the activation loop. EMBO J. 18:1309-1320.

19. Hilton, D.J., et al. 1998. Twenty proteins containing a C-terminal SOCS box form five structural classes. Proc. Natl. Acad. Sci. USA 95:114-119.

20. Masuhara, M., et al. 1997. Cloning and characterization of novel CIS family genes. Biochem. Biophys. Res. Commun. 239:439-446.

21. Auernhammer, C.J., Bousquet, C., and Melmed, S. 2000. Autoregulation of pituitary corticotroph SOCS-3 expression: characterization of the murine SOCS-3 promoter. Proc. Natl. Acad. Sci. USA. 96:6964-6969.

22. Sakamoto, H., et al. 1998. A Janus kinase inhibitor, JAB, is an interferongamma-inducible gene and confers resistance to interferons. Blood. 92:1668-1676.

23. Hamanaka, I., et al. 2001. Induction of JAB/SOCS-1/SSI-1 and
CIS3/SOCS-3/SSI-3 is involved in gp130 resistance in cardiovascular system in rat treated with cardiotrophin-1 in vivo. Circ. Res. 88:727-732.

24. Sasaki, A., et al. 2000. CIS3/SOCS-3 suppresses erythropoietin (EPO) signaling by binding the EPO receptor and JAK2. J. Biol. Chem. 275:29338-29347.

25. Nicholson, S.E., et al. 2000. Suppressor of cytokine signaling-3 preferentially binds to the SHP-2-binding site on the shared cytokine receptor subunit gp130. Proc. Natl. Acad. Sci. USA. 97:6493-6498.

26. Marine, J.C., et al. 1999. SOCS3 is essential in the regulation of fetal liver erythropoiesis. Cell. 98:617-627.

27. Rockman, H.A., et al. 1991. Segregation of atrial-specific and inducible expression of an atrial natriuretic factor transgene in an in vivo murine model of cardiac hypertrophy. Proc. Natl. Acad. Sci. USA. 88:8277-8281.

28. Kanegae, Y., et al. 1995. Efficient gene activation in mammalian cells by using recombinant adenovirus expressing site-specific Cre recombinase. Nucleic Acids Res. 23:3816-3821.

29. Hanakawa, Y., Amagai, M., Shirakata, Y., Sayama, K. and Hashimoto, K. 2000. Different effects of dominant negative mutants of desmocollin and desmoglein on the cell-cell adhesion of keratinocytes. J. Cell Sci. 113:1803-1811.

30. Wang, Y., et al. 1998. Cardiac muscle cell hypertrophy and apoptosis induced by distinct members of the p38 mitogen-activated protein kinase family. J. Biol. Chem. 273:2161-2168.

31. Hoshijima, M., Sah, V.P., Wang, Y., Chien, K.R., and Brown, J.H. 1998. The low molecular weight GTPase Rho regulates myofibril formation and organization in neonatal rat ventricular myocytes. Involvement of Rho kinase. J. Biol. Chem. 273:7725-7730.

32. Kunisada, K., et al. 2000. Signal transducer and activator of transcription 3 in the heart transduces not only a hypertrophic signal but a protective signal against doxorubicin-induced cardiomyopathy. Proc. Natl. Acad. Sci. USA. 97:315-319.

33. Marine, J., et al. 1999. SOCS1 deficiency causes a lymphocyte-dependent perinatal lethality. Cell. 5:609-616.

34. Alexander, W.S., et al. 1999. SOCS1 is a critical inhibitor of interferon gamma signaling and prevents the potentially fatal neonatal actions of this cytokine. Cell. 5:597-608.

35. Kuwahara, K., et al. 1999. Involvement of cardiotrophin-1 in cardiac myocyte-nonmyocyte interactions during hypertrophy of rat cardiac myocytes in vitro. Circulation. 100:1116-1124

36. McKinsey, T.A., and Olson, E.N. 1999. Cardiac hypertrophy: sorting out the circuitry. Curr. Opin. Genet. Dev. 9:267-274.

37. Rothermel, B.A., et al. 2001. Myocyte-enriched calcineurin-interacting protein, MCIP1, inhibits cardiac hypertrophy in vivo. Proc. Natl. Acad. Sci. USA. 98:3328-3333.

38. Yang, J., et al. 2000. Independent signals control expression of the calcineurin inhibitory proteins MCIP1 and MCIP2 in striated muscles. Circ. Res. 87:E61-E68.

39. Bueno, O.F., et al. 2001. The dual-specificity phosphatase MKP-1 limits the cardiac hypertrophic response in vitro and in vivo. Circ. Res. 88:88-96. 